\title{
Assessment of pupillary light reflex using a smartphone application
}

\author{
YOUNG DUCK SHIN ${ }^{1}$, JIN HO BAE ${ }^{1}$, EUN JUNG KWON ${ }^{1}$, \\ HYEON TAE KIM ${ }^{1}$, TAE-SOO LEE ${ }^{2}$ and YOUNG JIN CHOI ${ }^{3}$ \\ ${ }^{1}$ Department of Anesthesiology and Pain Medicine; ${ }^{2}$ Department of Biomedical Engineering; ${ }^{3}$ Department of Surgery, \\ College of Medicine, Chungbuk National University, Cheongju, Chungcheongbuk-do 361-763, Republic of Korea
}

Received March 30, 2015; Accepted April 29, 2016

DOI: $10.3892 /$ etm.2016.3379

\begin{abstract}
In unconscious patients, pupillary light reflex is an indicator of brain damage. In the current study, a smartphone application was developed for the purpose of measuring pupillary light reflex with an aim to determine the agreement between pupillary light reflex measurements using a smartphone application (APP) and a penlight (PEN). The APP acquires five sequential photographs using the camera flash in order to stimulate the pupil. The initial image is captured prior to the flash, and the subsequent image is obtained while the flash is on. The remaining three images are captured whilst the flash is off. Pupillary right reflex was assessed in 30 healthy subjects using a PEN. After $10 \mathrm{~min}$, the examiners inspected the images of light reflex acquired from the same subjects using the APP, and completed the corresponding questionnaire containing details of pupil size and degree of response. Agreement between the two assessment methods was determined by calculating bias, limits of agreement, and the intraclass correlation (ICC) coefficient. A statistically significant difference was not observed between the two methods regarding pupil size and degree of response. Bias was $0.1 \mathrm{~mm}$ and limits of agreement were $\pm 1.5 \mathrm{~mm}$, as compared with PEN. ICC was 0.93 (95\% confidence interval, 0.89-0.96). Therefore, it may be concluded that the results of pupillary light reflex assessed by PEN and APP display no significant difference. Furthermore, the APP provides advantages such as portability, objectivity and the possibility of being used as objective medical evidence.
\end{abstract}

Correspondence to: Professor Jin Ho Bae, Department of Anesthesiology and Pain Medicine, College of Medicine, Chungbuk National University, 52 Naesudong-Ro, Seowon-Gu, Cheongju, Chungcheongbuk-do 361-763, Republic of Korea

E-mail: yydshin@naver.com

Key words: cell phone, mobile application, pupillary reflex

\section{Introduction}

Pupillary light reflex is an important indicator for the determination of whether an unconscious patient's brain is damaged $(1,2)$. Pupillary light reflex should be assessed to determine the therapy the patient is to receive, and to evaluate the patient's status prior to and subsequent to treatment. In addition, if medical disputes arise, obtaining objective data regarding pupillary light reflex, which reflects intracranial status that is directly connected to patient's life, is necessary.

With the use of a pupillometer, a method by which to measure pupillary light reflex, an accurate measurement of the size of the pupil may be obtained. However, its use is accompanied by constraints, such as a lack of portability and usability (3). The most common method for the measurement of pupillary light reflex utilizes a penlight (PEN) (4). PENs are cost-effective and portable. However, by using a PEN, quantitative assessment is difficult to achieve as a result of its dependence upon the subjective judgment of a clinician and their level of experience.

Smartphones have received attention due to their use as an easy tool by which to produce and utilize information for the development of applications (5), and continuous development is possible by updating APPs. An APP was developed in the current study by utilizing portable smartphones to measure pupillary light reflex easily and conveniently. The aforementioned APP was designed to obtain objective results and a quantitative interpretation by measuring pupillary light reflex using the camera and flash function available on smartphones. The present study aimed to explore whether pupillary light reflex measured by using two methods, a smartphone APP and PENs, displayed significant differences, in addition to identifying the potential for the use of an APP as a basis for further studies.

\section{Materials and methods}

Development of a smartphone application. The Samsung Galaxy Series of smartphones were used in the present study. The camera flash was utilized to provide light stimulation to the pupil and acquire images to assess the pupils' reaction to the stimulation. In the current study, a Galaxy S4 (Samsung; Gyeonggi-do, South Korea) was used. As presented in Fig. 1, 
the main interface opens once the APP is run. The camera mode was activated using the touch panel, and the 'shot' button was subsequently used to implement pupillary light reflex examination. One image was captured prior to light stimulation, another image was acquired during the light stimulation, and several images were obtained at various time intervals after the flash had been turned off (Fig. 2). During the development of this APP protocol, the initial settings for acquiring images were set as 5 pictures in $6 \mathrm{sec}$, with focus set to 'close-up' and the optical image stabilization function enabled. Exposure was set to the maximum to ensure that the border between the iris and the pupil could be clearly distinguished.

Captured images were sent to the touch panel through a graphic processor (5.0-inch full HD super AMOLED display; Samsung). Results were displayed on the screen. On the left hand side, the initial image captured prior to light stimulation is presented; whereas the four images obtained following the light stimulation are displayed on the right hand side of the screen, to enable comparison (Fig. 3). Images were stored on the smartphone with the date and time so they could be viewed again at a later time-point.

Subjects and methods. The present study was approved by the Institutional Review Board of Chungbuk National University Hospital (approval no. 2014-10-003), and written informed consent was obtained from each volunteer. Pupillary light reflex was measured using a PEN (3 M LED FL-3000 Plus, 3 M Company, Maplewood MN, USA) and an APP on 30 healthy volunteers. Volunteers included 18 women and 12 men, aged 33.1 \pm 5.3 years (range, 24-49 years), of Korean descent. Assessment was performed in a room with fluorescent light (illuminance, 200-230 lux). Volunteers sat on a chair with their upper body tilted $30^{\circ}$ and measurements commenced once the patient's pupils had stabilized with their eyes closed for $\sim 6$ min. Assessors opened the eyelids of volunteers; therefore, their eyes were opened passively. Volunteers were instructed to look straight ahead rather than focus on certain areas. When using the PEN, the eyelid of the volunteer was opened with one hand and the penlight was held 10-15 cm away from the eye with the assessor's other hand. When using the APP, assessors held the camera lens in front of volunteers' eye at a distance of $15 \mathrm{~cm}$ and the volunteers' eyelid was opened with the remaining hand. The hand holding the camera was used to press the 'shot' button on the screen.

One doctor, who is familiar with measuring pupillary light reflex, conducted pupillary light reflex examination with a PEN, and pupil size and degree of response were recorded. Volunteers were permitted to rest for $10 \mathrm{~min}$ with their eyes closed. Following this, another tester used the APP to obtain images. In the same manner, pupillary light reflex data for all 20 volunteers were gathered using the two respective methods. After all data were obtained, the doctor who previously used the PEN recorded the pupil size and degree of response using only the images from the APP. During this period, the doctor did not receive any data retrieved from the PEN method. Upon completion of these processes, a comparative analysis of the data retrieved from the PEN and the APP was performed.

Pupil size (in mm) and degree of response were assessed on the checklist. Initial pupil size was recorded prior to light stimulation and minimum pupil size was recorded following

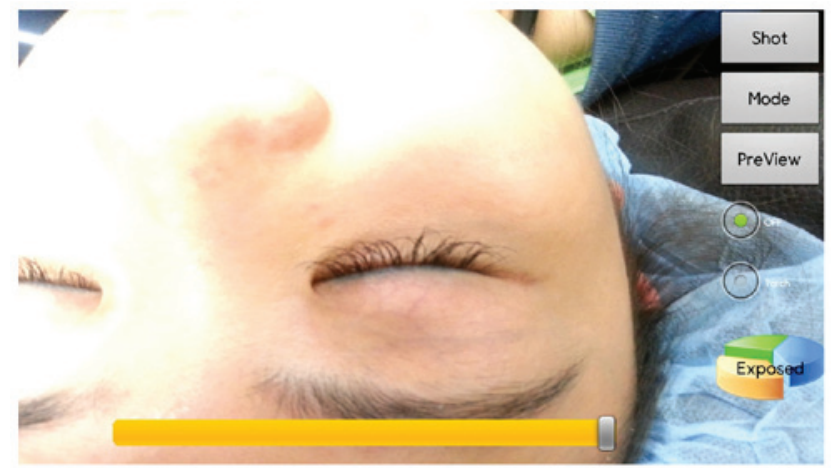

Figure 1. Main interface of the application upon opening.

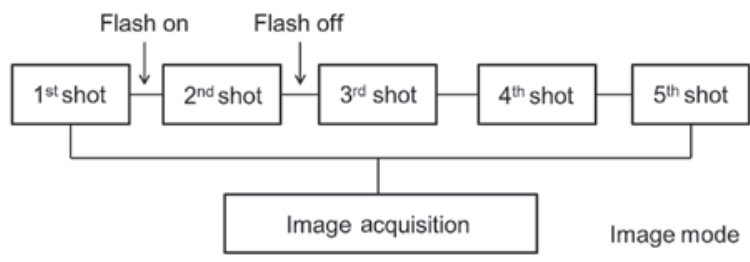

Figure 2. Representative schematic of the application protocol.

light stimulation. A pupil size scale diagram was provided to ensure that assessors were able to measure the size objectively (4). For degree of response, recorders selected from the following subjective indicators: Prompt $(++)$; sluggish $(+)$; and no response (-). Value of $\kappa$ was also determined, and was categorized as follows: $<0.20$, poor; $0.21-0.40$, fair; $0.41-0.60$, moderate; $0.61-0.80$, good; and $0.81-1.00$, very good.

Statistical analysis. Statistical analyses were performed using SPSS software, (version 12.0; SPSS, Inc., Chicago, IL, USA). Paired t-test was used to compare initial pupil size measured by the two methods, and to compare pupil size following light stimulation measured by the two methods. Paired t-test and the Bland-Altman method were used to estimate the bias and limits of agreement between the two techniques for the estimation of pupil size. Agreement was measured using the intraclass correlation (ICC) coefficient. In addition, degree of response measured by the two techniques yielded a discontinuous value, and cross tabulation was used to assess concordance. A $\kappa$-value $>0.8$ was considered to indicate good consistency. $\mathrm{P}<0.05$ was considered to indicate a statistically significant difference.

\section{Results}

Pupil size. From the subjects of the current study $(n=30)$, 60 paired comparisons were obtained. Images of pupillary light reflex acquired from the APP are presented in Fig. 3. Initial pupil size was $6.0 \pm 1.9 \mathrm{~mm}$ when measured using a PEN, and $5.8 \pm 1.8 \mathrm{~mm}$ when measured by the APP. There was no significant difference between the two methods regarding initial pupil size. However, on average, initial pupil size was greater when measured by the PEN, as compared with the APP. Furthermore, pupil size following light stimulation was $2.9 \pm 1.1 \mathrm{~mm}$ for the PEN and $2.8 \pm 1.0 \mathrm{~mm}$ for the APP, with no significant difference detected (Table I). 
Table I. Comparison of pupil size prior to and following light stimulus.

\begin{tabular}{|c|c|c|c|}
\hline Variable & Stimulus tool & Pre-light stimulus (mm) & Post-light stimulus (mm) \\
\hline \multirow[t]{2}{*}{ Pupil size (mm) } & Pen light & $6.0 \pm 1.9$ & $2.9 \pm 1.1$ \\
\hline & Smartphone application & $5.8 \pm 1.8$ & $2.8 \pm 1.0$ \\
\hline P-value & & 0.083 & 0.293 \\
\hline
\end{tabular}

Data are presented as the mean \pm standard deviation.

Table II. Bland-Altman analysis and interclass correlation coefficient.

\begin{tabular}{lccc}
\hline Variable & Bias $(\mathrm{mm})$ & Limits of agreement $(\mathrm{mm})$ & Intraclass correlation coefficient \\
\hline 60 comparisons & $0.1(\mathrm{P}=0.33)$ & \pm 1.5 & $0.93(95 \% \mathrm{CI} \mathrm{0.89-0.96)}$ \\
\hline
\end{tabular}

CI, confidence interval.

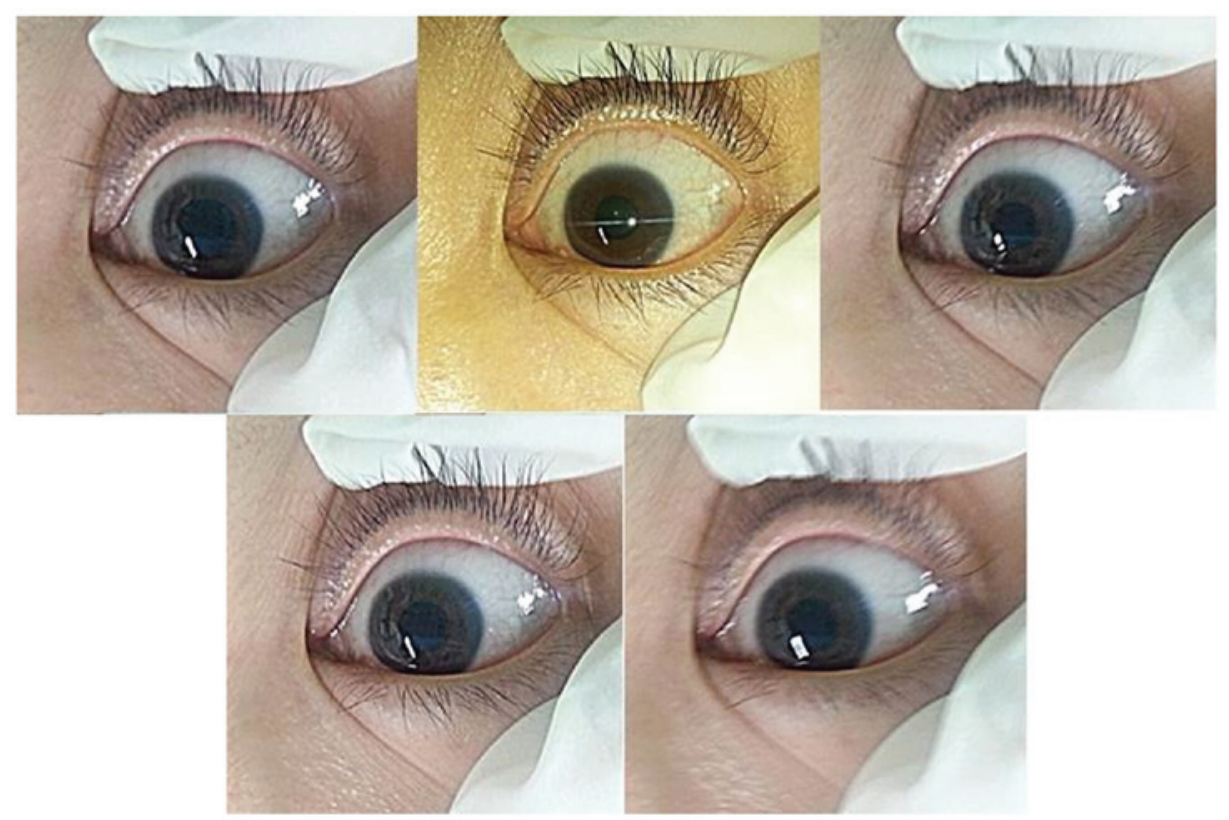

Figure 3. Five sequential images displaying pupillary light reflex indicated a normal response.

Bias and limits of agreement. The Bland-Altman method was used to estimate the bias and limits of agreement between the two techniques for measuring pupil size (Fig. 4). Bias was $0.1 \mathrm{~mm}$ and limits of agreement were $\pm 1.5 \mathrm{~mm}$ when the APP was compared with the PEN. ICC was 0.93 (95\% confidence interval, 0.89-0.96) (Table II). Degree of response of pupillary light reflex between the two methods indicated a high consistency, with a $x$-value of 1.00 (Table III).

\section{Discussion}

Assessment of pupillary light reflex should be performed under as follows: In a slightly dark environment, with patients looking far away, and assessors should observe the contraction of each pupil by flashing light onto one eye. In order to avoid a miosis effect due to the near reflex, pupillary light reflex should be assessed by instructing patients to fix their focus upon a particular object some distance away (6). However, as the current experiment aimed to compare the use of the two techniques in emergency situations involving unconscious patients, a similar situation as observed when examining patients in the emergency room or intensive care unit, was simulated. This was conducted by instructing volunteers to lay down under fluorescent light. If patients were to open and close their eyes constantly, their pupils may have constricted in response to the fluorescent light. Also, near reflex may occur when patients consciously stare at the camera flash or the tip of the PEN. To avoid these two phenomena, the patients' eyes were held open. In the current study, no significant difference in initial pupil size was detected between the two methods; however, on average, initial pupil size was greater when measured by the PEN, as compared with the APP. 
Table III. Cross-tabulation of pupillary light reflex.

\begin{tabular}{lcccr}
\hline & \multicolumn{2}{c}{ Smartphone application } & \\
\cline { 2 - 3 } & Prompt & Sluggish & No response & Total \\
\hline Pen light & & & & \\
Prompt & 30 & 0 & 0 & 30 \\
Sluggish & 0 & 0 & 0 & 0 \\
No response & 0 & 0 & 0 & 0 \\
Total & 30 & 0 & 0 & 30 \\
Value of $\kappa$ & 1.00 & & & \\
\hline
\end{tabular}

We hypothesize that this is because pupil size was assessed immediately after the patients' eyes were opened when the PEN was used. In contrast, when the APP was used, during the time taken to open the patient's eyes, operate camera, and capture the images, the patient's pupil may have contracted in response to the lights. A 10 -min rest was employed between the two techniques due to the consideration of re-expansion time of the pupil following the execution of pupillary light reflex. In a study concerning pupil re-expansion conducted by Léon et al (7), 14 healthy subjects were treated with red and blue light stimulation. Upon the administration of red light stimulation, it took $11 \mathrm{sec}$ on average for the patients' pupil to return to $90 \%$ of the initial size, and after undergoing blue light stimulation, it took $16 \mathrm{sec}$ on average. In addition, it took 17 and $22 \mathrm{sec}$ for the patients' pupil to return to $95 \%$ of their initial size following stimulation with red and blue light, respectively. From the results of this previous study (7), we hypothesize that $10 \mathrm{~min}$ after pupillary light reflex assessments, pupil size would have returned to the initial size. In the present study, a pupil size scale diagram was used to improve objectivity when completing a checklist after measuring pupil size. When using the PEN, the checklist was primarily conducted from memory. By contrast, when using the APP, details were assessed more objectively due to the ability to compare images and pupil size with the scale diagram. However, in the images obtained by a smartphone, pupil size could be exaggerated as a result of close-up filming. To adjust for this, pupil size was determined by comparing it with the size of the entire eye, and the pupil size scale diagram was used for reference alone. Due to the aforementioned reasons, we suggest that some error in size may have arisen.

Following the administration of light stimulation, pupil contraction occurs after a pupil incubation period due to the slow action of the iris muscle (8). The range of pupil incubation period is $180-500 \mathrm{msec}$, which decreases with increasing light intensity. Furthermore, in normal cases, the range increases by $1 \mathrm{msec}$ with age; $235 \mathrm{msec}$ at the age of 20 years old and $280 \mathrm{msec}$ when one is 70 years old $(9,10)$. As pupil incubation occurs within $300 \mathrm{msec}$, and the speed of pupil contraction is most rapid at the beginning, the minimal pupil size after pupillary light reflex was determined from the second image captured after the flash is enabled. When the flash is enabled, miosis is maintained and immediately after the light is turned off, the pupil begins to return to the initial pupil state. Pupil expansion speed is fastest the moment

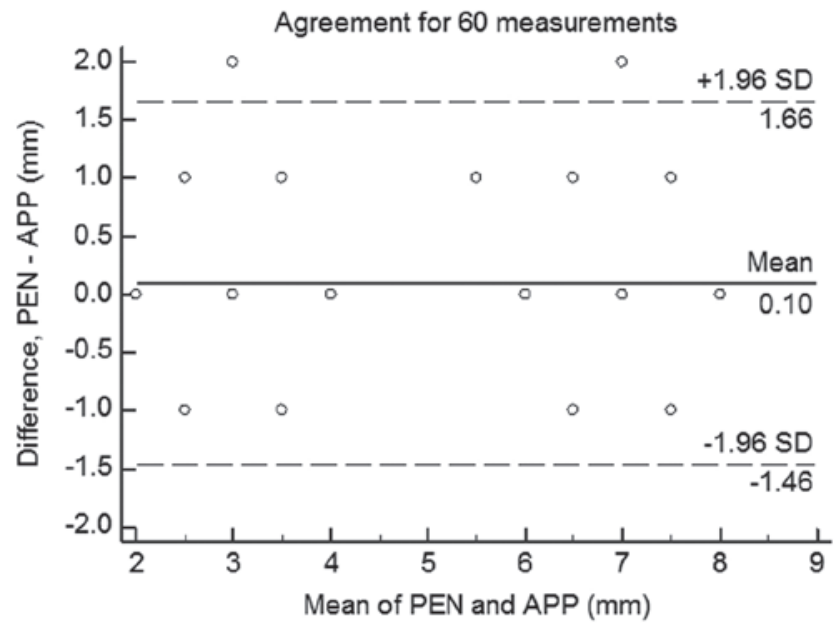

Figure 4. Bland-Altman plot for 60 paired measurements. Reference lines (broken lines) indicate the mean difference between the two methods (bias) at $0.1 \mathrm{~mm}$ and the $95 \%$ limits of agreement (1.7 to $-1.5 \mathrm{~mm}$ ).

the light is removed (8). Therefore, from the third image, the image captured after the flash is turned off, an image of the expanded pupil size was obtained. In this manner, as five consecutive images were compared, the aspect of transition was obtained in a stream of time, and pupillary light reflex response was accurately determined.

The major limitation of the APP in the present study was obtaining a clear image. When capturing an image using a smartphone, the same subject may appear differently according to light surroundings. Particularly, it is difficult to classify the pupil from the iris as Asians typically have a dark iris color. For accurate measurement, researchers have previously used continuous infrared illumination and an infrared-sensitive camera (10). As this method could not be applied on smartphones due to its complexity and the substantial costs involved, the current study upgraded the application setting to adjust the exposure in order to expose the boundary of the pupil as clearly as possible. Once exposure was set to the maximum, allowing the subjects to be clearly visualized in good light, satisfactory images were obtained.

The current study investigated pupillary light reflex measured by a PEN and an APP, and the results indicated that pupil size had no significant difference and pupil degree of response was high in consistency between the two methods. However, there were a number of limitations to the present study. Research was conducted on a limited patient group as the current study was unable to recruit patients who displayed a sluggish pupil response. In addition, the sample size was small and was not divided into age, gender or profession. With the use of various patient groups and upgrading the APP to ensure it was easier to use, the present application may be useful in measuring pupillary light reflex. In addition, if more objective data could be obtained by developing an application that was able to automatically measure pupil size or determine pupil contraction degree, this would have substantial benefits. The APP could be used for tracking observations during the patient treatment process, obtaining objective medical evidence, constructing a report structure among medical personnel, and aiding telemedicine systems 
as a form of supplementary data. The aforementioned benefits will contribute to changes and developments within the field.

\section{Acknowledgements}

The current study was supported by a research grant from Chungbuk National University in 2013.

\section{References}

1. Santhanam R, Pillai SV, Kolluri SV and Rao UM: Intensive care management of head injury patients without routine intracranial pressure monitoring. Neurol India 55: 349-354, 2007.

2. Meyer S, Gibb T and Jurkovich GJ: Evaluation and significance of the pupillary light reflex in trauma patients. Ann Emerg Med 22: 1052-1057, 1993.

3. Matouskova O, Slanar O, Chytil L and Perilk F: Pupillometry in healthy volunteers as a biomarker of tramadol efficacy. J Clin Pharm Ther 36: 513-517, 2011.
4. Jonathan $\mathrm{CH}$ : Disorders of the eye In: Harrison's Principles of Internal Medicine. Longo DL, Fauci AS, Kasper DL, Hauser SL, Jameson JL and Loscalzo J (eds). Vol I, 18th edition. McGraw-Hill, New York, pp224-225, 2012.

5. Zvornicanin E, Zvornicanin J and Hadziefendic B: The use of smart phones in opthalmology. Acta Inform Med 22: 206-209, 2014.

6. Jonathan CH: Disturbances in vision and ocular movements In: Harrison's Principles of Internal Medicine. Longo DL, Fauci AS, Kasper DL, Hauser SL, Jameson JL and Loscalzo J (eds). Vol I, 13th edition. McGraw-Hill, New York, pp101, 1994.

7. Léon L, Crippa SV, Borruat FX and Kawasaki A: Differential effect of long versus short wavelenghth light exposure on pupillary re-dilation in patients with outer retinal disease. Clin Experiment Ophthalmol 40: e16-e24, 2012.

8. Ellis CJ: The pupillary light reflex in normal subjects. Br J Ophthalmol 65: 754-759, 1981.

9. Bergamin O and Kardon RH: Latency of the pupil light reflex. Sample rate, stimulus intensity and variation in normal subjects. Invest Ophthalmol Vis Sci 44: 1546-1554, 2003.

10. Whiting RE, Yao G, Narfström K, Pearce JW, Coates JR Dodam JR, Castaner LJ and Katz ML: Quantitative assessment of the canine pupillary light reflex. Invest Ophthalmol Vis Sci 54: 5432-5440, 2013. 